\title{
Error Producing Conditions in the Intensive Care Unit
}

\author{
Frank A. Drews, PhD; Adrian Musters, BS; Matthew H. Samore, MD
}

\section{Abstract}

Up to 98,000 patients die because of human error in U.S. hospitals each year. Among the areas where errors occur frequently is the intensive care unit (ICU). Despite the impact of these errors, little research has identified the human factors that contribute to errors in the ICU. The current study uses the error-producing conditions (EPC) approach to help identify device-related factors that contribute to error. One result of the present study was the identification of the extent to which individual conditions contribute to the prevalence of error. In addition, we identified the contribution of certain devices to the prevalence of error. More importantly, the most critical devices for patient care were also identified as the devices that were rated highest in EPC prevalence. Developing medical devices designed to reduce the device-related potential for patient harm should be a primary goal in patient safety.

\section{Introduction}

Up to 98,000 patients die because of human error in U.S. hospitals each year. ${ }^{1}$ Critical care is one of the areas in which human errors occur most frequently. In their seminal paper, Donchin and colleagues ${ }^{2}$ estimated that the error rate was 1.7 per patient per day in the intensive care unit (ICU). A study 2 years later found that 45.8 percent of ICU admissions (480 of 1,047 ICU admissions) were associated with an adverse event (AE). ${ }^{3}$

More recently, an investigation of the incidence and nature of AEs and medical errors in a medical ICU and a coronary care unit (CCU) collected data during nine 3-week periods. A total of 120 AEs were found in 79 patients, for an AE rate of 20.2 percent. ${ }^{4}$ The researchers found that 66 of the 120 AEs (55 percent) were nonpreventable, while 54 of the AEs (45 percent) were preventable.

An Australian study suggested that nearly 17 percent of all hospital admissions resulted in an AE, with half of these being preventable. ${ }^{5}$ The same study estimated that in Australia, 8 percent of all hospital bed days were a result of AEs. In addition to the costs associated with a longer stay and other potential costs for the hospital, there were also the issues of disability payments and the associated high personal costs for the patients and staff involved in these cases.

More recently, due to a change in Medicare in the United States, the costs associated with AEs will be redistributed. Previously, the followup costs for treatment of "serious preventable events" were covered by insurance payments. However, as announced in September 2007, Medicare will no longer cover the followup costs of AEs. ${ }^{6}$ 


\section{Clinical Risk Management}

Historically, a human error in clinical settings has been treated as an issue of clinical risk management, the aim of which was to deal with the consequences of AEs by controlling the potential for litigation based on claims of medical negligence. ${ }^{7}$ One problem with this approach, though, was that each $\mathrm{AE}$ was treated as a single, isolated event. Underlying influences were not necessarily investigated, and the effects of other factors on the genesis of human error were often ignored. Consequently, errors were seen as an exception in a system that otherwise performed well; no other problems, other than the particular case in question, were typically investigated. Preventable AEs were considered to be the result of an individual making an error or not following standards of medical practice.

Several alternative approaches have been proposed and applied to improve our understanding of human error in health care. Among these is root cause analysis (RCA), a standard approach for the analysis and assessment of processes in the clinical environment. The Joint Commission requires this technique for studying serious and sentinel events. The goal of this resourceintensive method is to identify any aberrant processes that may have contributed to a particular AE. Root causes, which represent the fundamental reasons why the event occurred, are investigated by analyzing how and why the event occurred.

RCA has been criticized for several reasons. First, as its name suggests, RCA posits a single cause leading to an error, which often may be an oversimplification. In most cases, a complex chain of events, including a number of contributing factors, are found to lead to an incident or accident. In addition, RCA often focuses only on the reconstruction of a particular event and not on an understanding of what the event reveals about systemic problems in the health care organization in which it occurred. As a consequence, RCA may only help to prevent the repetition of similar events in the future, and it is uncertain whether it actually does reduce the risk of such events. Finally, RCA often directs its focus on events that are among the most serious, while ignoring events that may be considered less serious. In fact, though, a concentration on less serious AEs may help provide insight into systemic causes of error. Efforts to improve patient safety in health care should to go beyond an RCA to incorporate a psychologically based theory of human error in organizational settings.

An alternative to the approaches outlined above is to evaluate human error from a systemic perspective. This would not only document error occurrence, but it would also aim to understand the complex underlying factors that contribute to error in the context of health care. This can be distinguished from RCA's reactive approach because it applies a more proactive strategy of error management; its goal is to anticipate where and how errors may occur. At the core of a systemic approach is identification of conditions under which errors occur and implementation of measures that would protect against such error occurrence. Overall, a system-oriented approach that emphasizes proactive and pre-emptive error management is in accord with the concepts of preventive medicine and patient safety that lie at the core of public health. ${ }^{8}$

Adverse outcomes: The last link in a chain of events. Several authors have developed frameworks that include the elements outlined above. Here we discuss James Reason's model of organizational accidents. ${ }^{9}$ Central to Reason's approach is the assumption that an accident or an adverse outcome is the final link in a chain of events. This implies that it is important to consider 
the actions of the people involved and to analyze the conditions and organizational factors under which they were working that may have had an impact on the AE under investigation. Reason distinguishes between two elements present in cases of human error: active failures and latent conditions.

Active failures can refer to several different types of actions:

- Action slips - for example, selecting the wrong menu when programming an infusion pump.

- Lapses-for example, a nurse performs a structured task but does not use a checklist and, as a result, forgets an important step.

- Mistakes - for example, due to an incorrect understanding of a situation, an individual takes actions that may be performed successfully but do not solve the initial problem.

- Violations of operating practices, procedures, or standards-for example, not performing hand hygiene when indicated.

Latent conditions are the result of decisions that were made by people who do not operate at the “sharp end" but are more remote. ${ }^{10}$ Latent conditions can be created by management or senior clinicians with responsibility for decisions at the unit level. They also can originate with decisions that are made at a higher organizational level but have direct implications for the way work is performed by staff at the "sharp end." Latent conditions result in situations from which an active failure may lead to an AE. Common latent conditions include high workloads, insufficient training and supervision, inadequate equipment, or imperfect equipment maintenance.

Although Reason's approach ${ }^{9}$ provides a theoretical framework for human error, one potential problem is that it does not allow easy identification of influences that increase the probability of error at the level at which errors are likely to occur.

Human Error Assessment and Reduction Technique. An approach that overcomes this weakness is the Human Error Assessment and Reduction Technique (HEART). Developed by Jeremy Williams, HEART has been applied successfully in a wide range of industries. ${ }^{11}$ HEART provides a set of generic task types and lists error probabilities associated with these tasks. The probabilities for error are identified based on a comprehensive review of the literature on human performance and human factors. In addition, HEART provides a list of error-producing conditions (EPCs) that can affect both task performance and the potential to increase the probability of error when a specific task is performed in the presence of these conditions. Thus, EPCs can be thought of as additional factors that increase the probability of error. Williams conceptualized them as factors that have a consistent effect on human reliability. Thus, they introduce constant error that is nonvariable and therefore can be studied systematically.

Given the importance of EPCs, it is plausible that they should be an integral part of a comprehensive model of human error, including that of Reason (Figure 1). The strength of such an integrative approach is that it is well rooted in the psychological literature on human performance and human factors. Moreover, this integrated approach facilitates the identification of conditions and task characteristics that lead to estimates of the probability of error. A disadvantage of this approach, though, is that it requires a trained observer to visit work places 


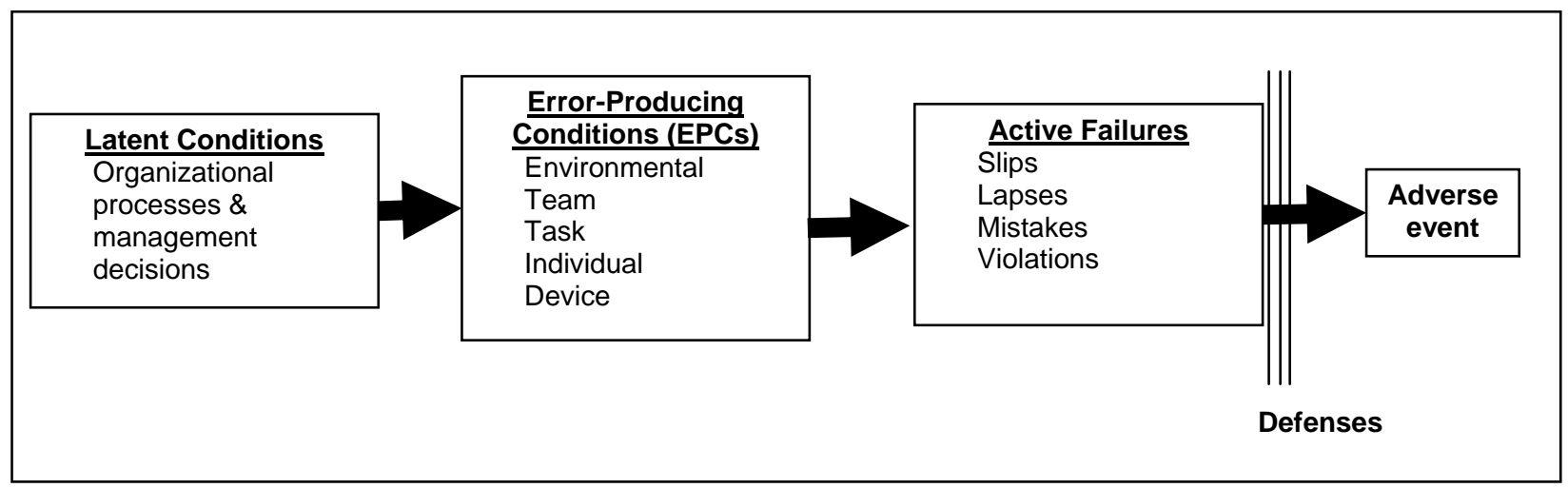

Figure 1. Reason's model of error (modified after Dean et al) ${ }^{14}$

under consideration and to categorize the tasks and conditions under which work is performed. Obviously, this makes the approach quite resource-intensive and time-consuming.

Most literature over the last three decades describes the application of systems like HEART in non-health care industries, demonstrating that proactive approaches toward error management have been successfully implemented. However, some encouraging evidence for the successful application of proactive and preemptive error management does come from health care. ${ }^{12}$

The present study focuses on EPCs in the ICU, which is a unique environment due to its complexity and tight coupling. (Coupling refers to the interdependence of different elements in a system.) The complexity of critical care medicine is reflected in the fact that high-risk decisions often have to be made by clinicians very rapidly, and to make matters worse, these decisions often are based on incomplete patient data. The presence of comorbidities in ICU patients only adds to the level of complexity, increasing the difficulty of decisionmaking even further. In addition, critical care patients often have lower physiologic reserves to help them cope with problems that might derive from suboptimal care.

Tight coupling presents other challenges. A tightly coupled system has little room for error because it cannot tolerate delays or variations in sequencing. Taken together, a high level of complexity and tight coupling make critical care medicine an ideal testing ground for the study of human error and the identification of EPCs.

EPCs in the ICU can be broadly considered as factors that negatively affect the reliability of human performance. The construct of EPCs is similar to the construct of latent causes of error, which has been extensively studied in non-health care industry settings, such as aviation and nuclear power plants. Although the notion that medical error arises from latent system-level causes is widely appreciated, ${ }^{13}$ quantitative data regarding the impact of these conditions in clinical settings are scant. ${ }^{14}$ To study EPCs, it seems important to focus either broadly on the presence of such conditions in the ICU, or to identify one or more specific domains and to examine their presence there. We have chosen the second approach and focused on devices that are associated with EPCs in the ICU. 


\section{Devices}

Potential problems with monitoring devices are highlighted by the following case: About 22 minutes after a nurse's last routine bedside visit, an ICU patient was found to be in torsades de pointes-type ventricular tachycardia (VT). As a result, the patient experienced irreversible brain damage. The staff noted that the critical arrhythmia alarm had not sounded at the onset of VT. Testing the monitoring equipment revealed that it had been inadvertently placed in "pulse mode," which disabled the arrhythmia alarms. Further investigation found that no critical care supervisors and only 3 of 50 staff nurses were even aware of the pulse mode option; none of the nurses knew of the pulse mode function's effect on alarms.

Sentinel event reports like this one provide much of the information about hazards associated with medical device use. The problem is that comprehensive studies of medical device problems are lacking. ${ }^{15}$ That alarms are used unproductively in ICUs is widely appreciated, yet very few data exist about the consequences of ineffective monitoring in critically ill patients.

Poor interface design in health care is a serious problem. The U.S. Food and Drug Administration (FDA) reported that from 1985 to 1989, up to 50 percent of device recalls were due to poor product design, including problems with software and user interfaces. ${ }^{16}$ As a result, in 1990, Congress passed the Safe Medical Devices Act (SMDA), which requires device producers to follow good manufacturing practices. ${ }^{17}$

In 2002, the Joint Commission issued an alert based on the review of 23 sentinel events involving mechanical ventilators, of which 19 had been associated with death and four with coma. ${ }^{18}$ Malfunction or misuse of an alarm or an inadequate alarm was a factor in 65 percent of the sentinel events; 52 percent were related to a tubing disconnect. The analysis of these ventilator events led the Joint Commission to designate improved effectiveness of clinical alarm systems as one of its patient safety goals.

Adapting the EPC framework based on Williams's work, we found it possible to identify a number of conditions that are directly related to the operation of medical equipment in the ICU. These EPCs, which formed the basis for our study, include:

1. Unfamiliarity with a situation.

2. Time pressure in error detection.

3. Low signal-to-noise ratio.

4. Mismatch between an operator's mental model and that imagined by the device designer.

5. Impoverished information quality.

6. Ambiguity in performance standards.

7. Disruption in normal work-sleep cycles.

8. Unreliable instrumentation.

The present study examines the prevalence of error-producing conditions, such as the ones above, with respect to the use of a selected number of medical devices in the ICU. The selection of specific devices was driven by their function (e.g., therapeutic or monitoring devices) and 
their criticality for patient safety (Table 1). ${ }^{19}$ In addition to EPCs, the study also assessed error prevalence in the ICU based on the subjective ratings of ICU nurses.

Historically, medical research has been focused more on the development of plans (i.e., identifying therapies) than on ensuring that those plans are correctly executed (i.e., therapeutic interventions). ${ }^{20}$ Focusing on nurses in this study gives us the opportunity to examine performance that is related to the execution of plans.

Based on a literature review, we expected to find that nurses would be able to identify the presence of errors in the ICU. Thus, we expected the presence of error in the ICU to receive moderate to high ratings. We also expected that nurses would be able to identify general EPCs and rate them in terms of their perceived contribution to ICU problems. However, no specific hypotheses were formulated about the ratings of individual EPCs. At least two hypotheses about the relationship between patient safety and EPCs, as they relate to a device's criticality, can now be developed:

- The higher a device’s criticality, the lower its rating for individual EPCs should be.

- Based on data about device use in the ICU, the higher the criticality of a device, the higher the rating of the impact of EPCs should be. ${ }^{19}$

\section{Methods}

The present study employed questionnaires (approved by the University of Utah Institutional Review Board) to determine nurses' assessments of ICU device-related EPCs. Nurse participants were recruited through advertisements placed at several ICUs in Salt Lake City, UT.

To be eligible for the study, nurses had to be active Registered Nurse license holders, have at least 1 year of experience in critical care, and be employed and currently working in an ICU. Nurses completed the questionnaires in the presence of a research assistant.

Data were collected from 25 ICU nurses. The mean age of participants was 39 years (range 2264 , sd $=13.5$ ), and they had an average of 10 years in critical care (range $1-38$, sd $=10.25$ ). The majority of participants were female (19), and most were currently working in the medical ICU (68 percent). Other intensive care workplaces included the thoracic ICU (12 percent), the surgical ICU (8 percent), the neurological critical care unit (8 percent), and the burn-trauma unit (4 percent). The demographics of these participants are shown in Table 2. 


\section{Materials}

To identify general and device-related error-producing conditions, we developed a questionnaire that focused on the eight EPCs mentioned above. The questionnaire consisted of 121 items that were grouped into three sections. Participants rated each statement on a scale from 1 ("strongly agree") to 9 ("strongly disagree"). After the individual scales were recoded to create unidirectional scales, mean scores across device categories were computed for each question.

The first section focused on errors in the ICU in general. Statements to assess this issue included: "Errors are a problem in the ICU"; "You sometimes find yourself making errors"; and others.

The second section provided statements about devices in general and how EPCs might affect operation of these devices. To assess participants' attitude about these EPCs, statements included "You are open and willing to ask for help when programming a device"; "You always can tell whether something is malfunctioning or just difficult to use"; and others.

In the third section, statements about specific EPCs had to be rated according to how they applied to individual devices that differed in criticality for patient safety (i.e., low, moderate, and high). Respondents were asked to rate their level of agreement with several device-specific comments, such as "You feel you have received adequate training to use [device]"; "You often see an error message with [device]"; "You feel [device] is designed well”; and others.

Respondents also had the option of not answering questions about individual devices if they had had no experience in using them.

After arriving at the Department of Psychology at the University of Utah (or another place of their choosing), nurses completed the consent form required by the University of Utah's Institutional Review Board. The nurses then completed the questionnaires, which took most nurses approximately 45 minutes.
Table 2. Demographics of participating

\begin{tabular}{lc}
\hline Total ICU nurses participating & 25 \\
\hline Sex & 6 \\
Male & 19 \\
$\quad$ Female & \\
\hline Age (years) & $39(14)$ \\
$\quad$ Mean ( \pm SD) & $22-64$ \\
$\quad$ Range & \\
\hline Years in critical care nursing & $10(10)$ \\
Mean ( \pm SD) & $1-38$ \\
Range & \\
\hline ICU workplace (\%) & 68 \\
Medical & 12 \\
Thoracic & 8 \\
Surgical & 8 \\
Neurological critical care & 3.8 \\
$\quad$ Burn-trauma unit & \\
\hline
\end{tabular}




\section{Results}

For the purpose of the analyses, the polarity of items was reversed where needed so that high ratings consistently expressed a more negative attitude toward an individual statement. Consequently, aggregated scores on individual scales (e.g., error scale) expressed more negative attitudes and had higher average ratings; i.e., this condition potentially contributes more to error occurrence.

The first analysis focused on participants' subjective assessments of errors occurring in their own behavior or observed in other ICU nurses. The mean $( \pm S D)$ rating on the scale that assessed the presence of error in the ICU was $6.35( \pm 1.3)$; range, 3.25 8.75. Mean EPC ratings in the ICU in general are shown in Table 3.

Clearly, the EPCs “low signal-to-noise ratio," “unreliable instrumentation,” “operator-designer mismatch," and "shortage of time” ranked relatively high in importance. These higher ratings contrasted with the nurses' ratings for "ambiguity in performance standards," which they rated as a less important contributor to error.

The final analysis focused on device-specific EPCs and the relationship between device criticality for patient safety and potential device hazards as contributors to error. For this purpose, we analyzed device-specific answers with regard to the EPCs using an ANOVA with device criticality (i.e., high, moderate, low) as a factor. Individual ANOVAs were performed for each of the eight EPCs.

The ANOVAs identified five device-specific EPCs with significant differences among the three criticality ratings (Table 4). The EPCs with significant differences were "unfamiliarity with situation" $[\mathrm{F}(2,71)=28.9 ; P$ $<0.01]$; "shortage of time" $[\mathrm{F}(2,71)=5.0 ; P<0.01]$; "low signal-to-noise ratio" $[\mathrm{F}$ $(2,71)=3.1 ; P<0.05)$; "operatordesigner mismatch" $[\mathrm{F}(2,71)=7.6$; $P<0.01)$; and "quality of information" [F $(2,71)=30.0$; $P<0.01$ ].
Table 4. Mean criticality ratings for statistically significant devicespecific EPCs

\begin{tabular}{lccc}
\hline & \multicolumn{3}{c}{ Mean (士SD) criticality rating } \\
\cline { 2 - 4 } $\begin{array}{l}\text { Device-specific error- } \\
\text { producing condition }\end{array}$ & High & Moderate & Low \\
\hline Unfamiliarity with situation & $4.4(2.9)$ & $2.3(1.7)$ & $1.3(0.5)$ \\
Shortage of time & $3.8(2.3)$ & $2.1(1.3)$ & $2.5(1.8)$ \\
Low signal-to-noise ratio & $3.3(2.1)$ & $2.1(1.2)$ & $3.1(1.8)$ \\
Operator-designer & $4.4(2.6)$ & $2.8(1.5)$ & $4.0(2.3)$ \\
mismatch & $4.0(2.6)$ & $2.0(1.0)$ & $4.0(2.4)$ \\
Quality of information & & & \\
\hline a ANOVA & & &
\end{tabular}


Figures 2 and 3 illustrate the mean agreement scores for "unfamiliarity" $\mathrm{x}$ device criticality rating and "shortage of time" $\mathrm{x}$ device criticality rating, respectively.

We performed additional post-hoc analyses to identify which differences in criticality levels for each of the above EPCs were statistically significant. With regard to the "unfamiliarity" variable, the mean rating for high device criticality was 4.4 vs. 2.3 for moderate criticality and 1.3 for low criticality (Table 4, Fig. 2). Tests indicated that differences among all three levels of device criticality were significant for the EPC "unfamiliarity with situation.” In clinical terms, this suggested that unfamiliarity with a device becomes less of a factor in the production of errors as the device's criticality declines.

Similarly, in the case of "shortage of time," data analyses indicated a significant difference between high and medium device criticality (mean rating $=3.8$ and 2.1, respectively) in the likelihood of causing an error; and between high and low device criticality (mean rating $=3.8$ and 2.5 , respectively); but there was

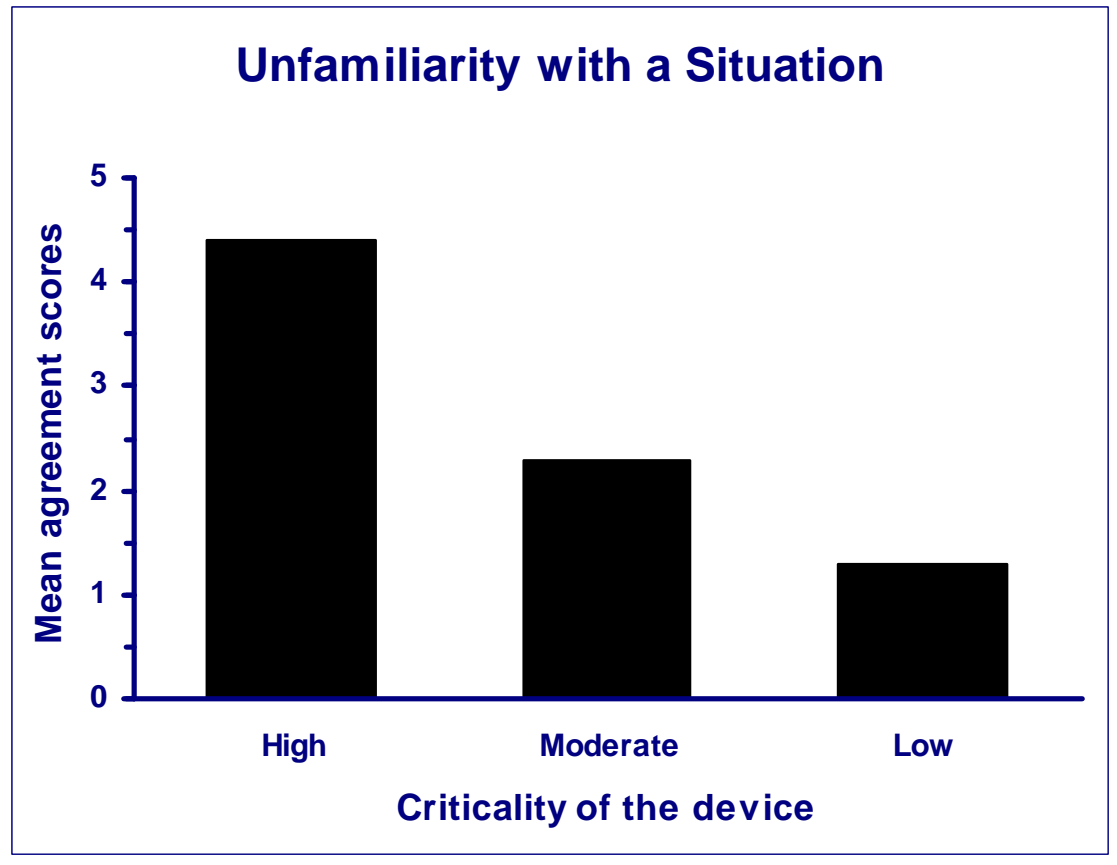

Figure 2. Mean agreement scores of device-specific EPCs for "unfamiliarity with a situation" $\mathrm{x}$ device criticality. Results suggest that "unfamiliarity" becomes less likely to lead to error as device criticality declines from high to low.

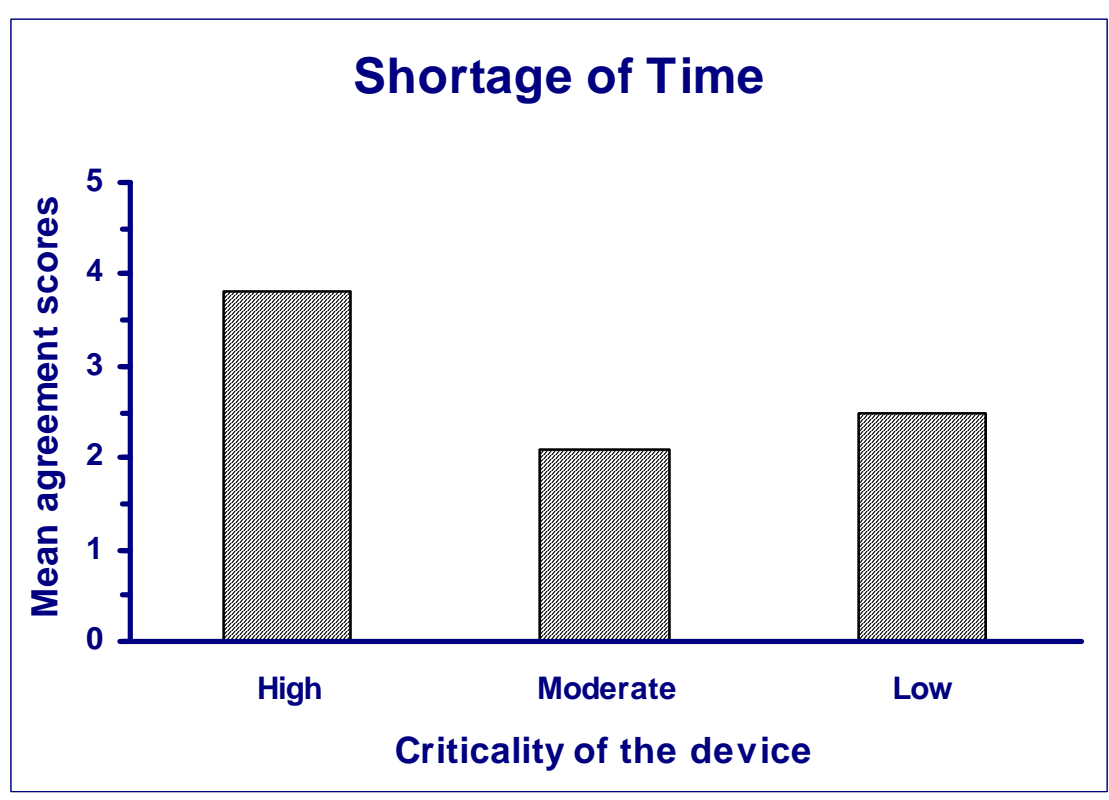

Figure 3. Mean agreement scores of device-specific EPCs for "shortage of time” x device criticality. Results suggest that shortage of time significantly increases the chance of error more with highly critical devices than with devices of moderate or low criticality. 
no significant difference between devices that were medium or low in criticality (Table 4, Figure 3). Thus, in clinical terms, the EPC "shortage of time" should have a significantly greater negative impact on the risk of errors when nurses are using highly critical devices compared with devices that are moderate or low in criticality.

In the case of the EPC “operator-designer mismatch,” significant differences in the risk of error were found between devices with high or moderate criticality (mean $=4.4$ and 2.8, respectively) and between devices with moderate or low criticality (mean $=4.4$ and 4.0 , respectively). For the EPC “quality of information,” significant differences were found between devices high or moderate in criticality (mean $=4.0$ and 2.0, respectively) and between devices moderate or low in criticality (mean $=2.0$ and 4.0, respectively). Interestingly, for both "operator-designer mismatch" and "quality of information," the risk of errors using high criticality devices and low criticality devices was similar when compared to those of moderate criticality.

For the EPC “low signal-to-noise ratio” no significant differences were found in a device's risk of errors based on its criticality

\section{Discussion}

The first goal of this study was to assess perception of error in the ICU among nurses who had experience working in the ICU. Another goal was to examine device-related EPCs in the ICU, based on the application of a survey methodology. Two aspects were analyzed: What are devicerelated EPCs, with no focus on specific devices; and how much are EPCs associated with devices according to their criticality?

Our initial finding is consistent with the existing literature on error in the ICU: Errors not only occur in the ICU, but they occur rather frequently, as reflected in the high mean nurse estimates of 6.35 (9-point scale).

A potential criticism of this finding is that the ratings were not especially high, and one might possibly expect higher subjective ratings. However, it is important to keep in mind that error in health care is an issue that clinicians do not necessarily like to discuss. Thus, it may be argued with equal validity that ICU error ratings even this high are rather remarkable. Often, when asking people to respond to statements that measure socially questionable acts or activities, the bias toward the lowest endpoint on a scale is strong. This tendency might even be amplified if people work in an organization marked by a culture of blame. Given that the present study found ratings on statements about error to be relatively high, this finding emphasizes the importance of investigating, in even greater detail, the nature of and contributing factors to error in the ICU.

Device-related EPCs in general shed some light on the contributing factors to error in the ICU. The results of the present study indicate that some EPCs are perceived as having a larger impact on error than others, when focusing on devices across the board. Clearly, low signal-to-noise ratio is one such EPC, indicating that information provided by devices in the ICU is difficult to discriminate from noise. This has serious implications for performance. Even experts experience serious difficulties performing at near optimal levels without interpretable and trustworthy feedback. A low signal-to-noise ratio has the potential to affect performance negatively. Some 
support for this finding comes from the observation of the large number of false alarms in the average ICU. ${ }^{2}$

Related to the problem of low signal-to-noise ratio is the issue of unreliable instrumentation. Nurses experience unreliable instrumentation when they have to deal with false sensor readings or other equipment-related problems that can cause false alarms. In another paper in the current edition of Advances in Patient Safety, we note that nurses point to incorrect sensor readings as one of the main problems of patient monitoring in the ICU. ${ }^{21}$

Another problem that ranks high as a potential contributing factor to error in the context of device use is "operator-designer mismatch" as it relates to the mental model implemented in the design of a device. Not only does the relatively high ranking of this EPC indicate it to be a potential hazard, but this finding is also consistent with nurses' complaints about cumbersome interfaces (e.g., patient monitors) that do not map into the task structure or sequence for which the device is used. ${ }^{21}$ Based on this finding, we can conclude that those involved in device development are not taking into adequate account cognitive analyses of the user's task and the device's interface. As a result, the device may not facilitate the task at hand as much as it increases the likelihood of the nurse committing an error.

Another EPC with high ratings was "shortage of time" in dealing with devices. Given the problems outlined above, it is no surprise to find that under time pressure, the operation of devices becomes more of a challenge and consequently increases the chance of an error.

Finally, the present study offers evidence about the relationship between EPCs and devices of varying criticality for patient safety. The results show that devices with high criticality unfortunately have the highest ratings on the EPCs "shortage of time" and "unfamiliarity with the situation." This finding replicates results from Samore and colleagues ${ }^{19}$ to a certain extent, but it also extends these by providing evidence that devices that are among the most important for patient safety also are the ones with the largest potential for patient hazards. Ironically, the use of these critical devices is especially compromised when nurses have to deal with significant challenges (e.g., unfamiliar situations) and are under high time pressure ("shortage of time”).

This particular finding has important implications for the development of devices and their interfaces: only ICU devices that minimize hazards and maximize resilience toward error have the potential to reduce the risk of human error. The development of devices that are consistent with these goals will be a formidable human factors task.

A potential limitation of the present study is the relatively small sample size (25 nurses). To increase the generalizability of our findings, future studies of EPCs in the ICU should employ larger sample sizes.

Based on the present findings, it appears that questionnaires - in addition to observational approaches used in prior non-health care domains — can be used to identify EPCs. By translating the construct "error-producing condition" into easily comprehensible language, it seems possible to collect data on these factors. Validation of these findings using independent observers is still 
important, though, because mismatches between a nurse's experience and the conclusions of a trained observer regarding potential EPCs cannot be excluded.

The overall results of this study are encouraging in that they demonstrate the possibility of increasing our understanding of device-related human error in the ICU. This is an important step toward the goal of reducing hazards and improving patient safety.

\section{Author Affiliations}

Department of Psychology, University of Utah, Salt Lake City, UT (Dr. Drews and Mr. Musters); Informatics, Decision-Enhancement, and Surveillance Center, VA Salt Lake City Health Care System, Salt Lake City, UT (Dr. Drews and Dr. Samore); Clinical Epidemiology, School of Medicine, University of Utah, Salt Lake City, UT (Dr. Samore).

Address correspondence to: Frank A. Drews, Department of Psychology, University of Utah, 380 S 1530 E, Rm 502, Salt Lake City, UT 84112; Email: frank.drews@psych.utah.edu.

\section{References}

1. Kohn LT, Corrigan JM, Donaldson MS, editors. To err is human: Building a safer health system. Washington, DC: National Academies Press; 2000.

2. Donchin Y, Gopher D, Olin M, et al. A look into the nature and causes of human errors in the intensive care unit. Crit Care Med 1995; 23: 294-300.

3. Andrews LB, Stocking C, Krizek T, et al. An alternative strategy for studying adverse events in medical care. Lancet 1997; 349: 309-313.

4. Rothschild JM, Landrigan CP, Cronin JW, et al. The critical care study: The incidence and nature of adverse events and serious medical errors in intensive care. Crit Care Med 2005; 33: 1694-1700.

5. Wilson RM, Runciman WB, Gibber RW, et al. The Quality in Australian Health Care Study. Med J Aust 1995; 163: 458-471.

6. Pear R. Medicare says it won't cover hospital errors. The New York Times, Aug. 19, 2007. Available at: www.nytimes.com/2007/08/19/washington/19hospital. $\mathrm{html}$ ?ei=5070\&en=6befd64203caa8ec\&ex $=11893104$ 00\&pagewanted=all. Accessed February 8, 2008.

7. Vincent CA, editor. Clinical risk management. London: BMJ Publications; 1995.

8. Stockwell DC, Slonim AD. Quality and safety in the intensive care unit. J Intensive Care Med 2006; 21: 199-210.
9. Reason J. Managing the risks of organizational accidents. Aldershot, UK: Ashgate; 1997.

10. Cook RI, Woods DD. Operating at the sharp end: The complexity of human error. In: Bogner MS, editor. Human error in medicine. Mahwah, NJ: Lawrence Erlbaum; 1994. p. 255-254.

11. Williams JC. A data based method for assessing and reducing human error to improve operational performance. Proceedings of IEEE Fourth Conference on Human Factors in Power Plants; 1988 5-9 June: Monterey, CA. p. 436-450

12. Carthey J, de Leval MR, Reason JT. The human factor in cardiac surgery: Errors and near misses in a high technology medical domain. Ann Thorac Surg 2001; 72: 300-305.

13. Vincent CA, Taylor-Adams S, Stanhope N. A framework for the analysis of risk and safety in Medicine. Br Med J 1998; 316: 1154-1157.

14. Dean B, Schachter M, Vincent C, Barber N. Causes of prescribing errors in hospital inpatients: A prospective study. Lancet 2002; 359: 1373-1379.

15. Xiao Y, Seagull J, Nieves-Khouw F, et al. Organizational-historical analysis of the "failures to respond to alarm" problem. IEEE transactions on systems, man, and cybernetics, Part A: Systems and humans. 2004; 34: 772-78. 
16. U.S. Food and Drug Administration. Center for Devices and Radiological Health. Human factors implications of the new Good Manufacturing Practices (GMP) rule and overall requirements of the new Quality System Regulation. Retrieved from http://www.fda.gov/cdrh/humfac/frqsr.html. Accessed February 26, 2008.

17. Safe Medical Devices Act (SMDA) of 1990. Public Law 101-629. Available at: http://legacy.library.ucsf.edu/tid/vrd22d00/pdf;jsessio nid=99779127FF9A3F647407CAA2582DB11D. Accessed February 26, 2008.

18. Joint Commission for the Accreditation of Healthcare Organizations. Preventing ventilator-related death and injuries. Sentinel Event Alert, Issue 25, February 26, 2002. Available at: www.jointcommission.org/SentinelEvents/SentinelEv entAlert/sea_25.htm. Accessed February 26, 2008.
19. Samore MH, Evans RS, Lassen A, et al. Surveillance of medical device-related hazards and adverse events in hospitalized patients. JAMA 2004; 291: 325-334.

20. Bates DW, Gawande AA. Patient safety: Improving safety with information technology. N Engl J Med 2003; 348: 2526-2534.

21. Drews FA. Patient monitors in critical care: Lessons for improvement. In: Advances in Patient Safety: New Directions and Alternative Approaches. Rockville, MD: Agency for Healthcare Research and Quality; 2008. 